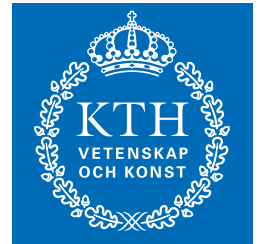 \\ KTH Electrical Engineering \\ Computational Framework for Optimal Robust Beamforming in Coordinated Multicell Systems
}

\author{
Best Student Paper Award \\ Proceedings of IEEE International Workshop on Computational Advances in \\ Multi-Sensor Adaptive Processing (CAMSAP).
}

13-16 December, San Juan, Puerto Rico, 2011

Copyright 2011 IEEE. Published in the IEEE 2011 International Workshop on Computational Advances in Multi-Sensor Adaptive Processing (CAMSAP 2011), scheduled for 13-16 December 2011 in San Juan, Puerto Rico. Personal use of this material is permitted. However, permission to reprint/republish this material for advertising or promotional purposes or for creating new collective works for resale or redistribution to servers or lists, or to reuse any copyrighted component of this work in other works, must be obtained from the IEEE. Contact: Manager, Copyrights and Permissions / IEEE Service Center / 445 Hoes Lane / P.O. Box 1331 / Piscataway, NJ 08855-1331, USA. Telephone: + Intl. 908-562-3966.

\section{EMIL BJÖRNSON, MATS BENGTSSON, GAN ZHENG, AND BJÖRN OTTERSTEN}

Stockholm 2011

KTH Royal Institute of Technology

ACCESS Linnaeus Center

Signal Processing Lab 


\title{
Computational Framework for Optimal Robust Beamforming in Coordinated Multicell Systems
}

\author{
Emil Björnson*, Mats Bengtsson*, Gan Zheng ${ }^{\dagger}$, and Björn Ottersten* ${ }^{*}$ \\ *Signal Processing Lab, ACCESS Linnaeus Center, KTH Royal Institute of Technology \\ ${ }^{\dagger}$ Interdisciplinary Centre for Security, Reliability and Trust (SnT), University of Luxembourg \\ Email: emil.bjornson@ee.kth.se; mats.bengtsson@ee.kth.se; gan.zheng@uni.lu; bjorn.ottersten@ee.kth.se
}

\begin{abstract}
Coordinated beamforming can significantly improve the performance of cellular systems through joint interference management. Unfortunately, such beamforming optimization problems are typically NP-hard in multicell scenarios, making heuristic beamforming the only feasible choice in practice. This paper proposes a new branch-reduce-and-bound algorithm that solves such optimization problems globally, with a complexity suitable for benchmarking and analysis. Compared to prior work, the framework handles robustness to uncertain intercell interference and numerical analysis shows higher efficiency.
\end{abstract}

\section{INTRODUCTION}

Most resource allocation problems in multiantenna multicell systems are non-convex and NP-hard [1], meaning that the optimal solution cannot be obtained in polynomial time. Thus, only heuristic suboptimal beamforming strategies can be applied in practice when trying to optimize, for example, the sum performance, proportional fairness, or harmonic mean. Still, it is very important to compute the optimal solution to use it as a benchmark and to study its properties.

The key to solve non-convex resource allocation problems systematically is to find a suitable parameter space that represents all feasible and some infeasible strategies, and then apply some global optimization technique to iteratively reduce this space with guaranteed convergence to a global optimum [2]. Recently, other authors have proposed spaces based on beamforming parametrizations [3], power allocation [4], user rates [5], and received interference [6]. The global optimization was either based on the polyblock approximation algorithm [3], [5] or the branch-and-bound algorithm [4], [6]. Out of these papers, only [5], [6] handles arbitrary multicell systems. In addition, all of these papers assume perfect channel state information (CSI) between all transmitters and all users in the system, which is almost impossible to achieve in practice.

Herein, we propose a framework for computing optimal coordinated beamforming with robustness to CSI uncertainty. We use a new branch-reduce-and-bound (BRB) algorithm and operate in the space of the robust performance region. At each iteration, we solve a quasi-convex resource allocation problem that we call robust fairness-profile optimization (RFO). We show by simulations that the BRB algorithm is more efficient than the polyblock approximation algorithm.

The research leading to these results has received funding from the European Research Council under the European Community's Seventh Framework Programme (FP7/2007-2013) / ERC Grant Agreement No. 228044.

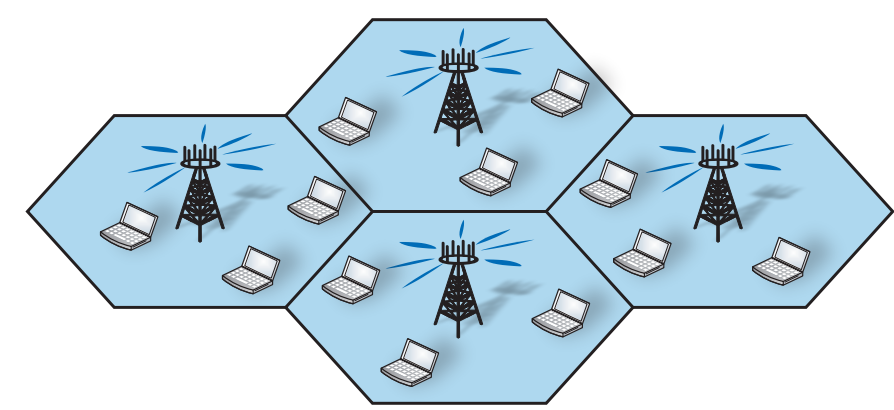

Fig. 1. A simple multicell system with $N=4$ cells and $K=3$ users per cell. Users are only served by their own base station, while interference is coordinated over the whole network. The CSI between cells is uncertain.

Note that each user is only served by its own cell and we only consider uncertainty towards out-of-cell interference. In our journal version [7], we take an alternative approach based on mean squared errors (MSEs) instead of signal-tointerference-and-noise ratios (SINRs), which enables general dynamic coordinated multipoint (CoMP) transmission and having joint uncertainty over channels from all base stations.

\section{SySTEM MODEL}

We consider a downlink multicell scenario with $N$ cells and $K$ users per cell. Each base station has $N_{t}$ antennas, while each user has a single antenna. This scenario is illustrated in Fig. 1. For simplicity, we adopt the notation of [8] where the received signal at the $j$ th user in the $i$ th cell is

$$
y_{i, j}=\sum_{l} \mathbf{h}_{i, i, j}^{H} \mathbf{w}_{i, l} x_{i, l}+\sum_{m \neq i, n} \mathbf{h}_{m, i, j}^{H} \mathbf{w}_{m, n} x_{m, n}+z_{i, j} .
$$

The scalar-coded data symbol to this user is $x_{i, j}$ and is zeromean and unit-variance, while $\mathbf{w}_{i, j} \in \mathbb{C}^{N_{t} \times 1}$ is the associated beamforming vector and $z_{i, j} \in \mathcal{C N}\left(0, \sigma^{2}\right)$ is white noise.

The channel vector from the base station in the $m$ th cell to the $j$ th user in the $i$ th cell is $\mathbf{h}_{m, i, j} \in \mathbb{C}^{N_{t} \times 1}$. In general, the exact value of $\mathbf{h}_{m, i, j}$ cannot be obtained by the system due channel estimation errors, feedback quantization, hardware deficiencies, feedback delays, small-scale fading, etc. It is common to model this uncertainty using an additive error model [7], [9], [10] with

$$
\mathbf{h}_{m, i, j}=\widehat{\mathbf{h}}_{m, i, j}+\boldsymbol{\epsilon}_{m, i, j}
$$

where $\widehat{\mathbf{h}}_{m, i, j} \in \mathbb{C}^{N_{t} \times 1}$ is the uncertain CSI obtained by the system and $\boldsymbol{\epsilon}_{m, i, j} \in \mathbb{C}^{N_{t} \times 1}$ is the error vector. The 
transmission cannot account for any error, which is handled by defining robustness towards a subset of error vectors, the uncertainty set, that either is defined probabilistically [11] or in a worst-case manner [7], [9], [10]. Herein, we adopt the latter approach since it can give exact convex problem formulations. We concentrate on (compact) ellipsoidal uncertainty sets

$$
\mathcal{U}_{m, i, j}=\left\{\mathbf{h}_{m, i, j}=\widehat{\mathbf{h}}_{m, i, j}+\mathbf{B}_{m, i, j} \tilde{\boldsymbol{\epsilon}}_{m, i, j}:\left\|\tilde{\boldsymbol{\epsilon}}_{m, i, j}\right\|_{2} \leq 1\right\}
$$

where $\mathbf{B}_{m, i, j} \in \mathbb{C}^{N_{t} \times N_{t}}$ defines the shape of the ellipsoid. Thus, the combined CSI at the transmitter-side is $\left\{\mathcal{U}_{m, i, j}\right\}$. This paper considers the case when the intracell channels are known (i.e., $\mathbf{B}_{m, i, j}=\mathbf{0}$ and $\mathbf{h}_{m, i, j}=\widehat{\mathbf{h}}_{m, i, j}$ for all $m=i$ ) and concentrates on robustness towards intercell interference. This makes sense since intercell CSI is harder to obtain. The general case when all channels are uncertain is solved in [7].

The $i$ th cell is subject to $L_{i}$ transmit power constraints

$$
\sum_{l} \mathbf{w}_{i, l}^{H} \mathbf{Q}_{i, k} \mathbf{w}_{i, l}=\operatorname{tr}\left\{\mathbf{W}_{i}^{H} \mathbf{Q}_{i, k} \mathbf{W}_{i}\right\} \leq q_{i, k} \quad \forall k
$$

where $\mathbf{W}_{i}=\left[\mathbf{w}_{i, 1} \ldots \mathbf{w}_{i, K}\right]$ is the combined beamforming matrix in the $i$ th cell. All $\mathbf{Q}_{i, k} \in \mathbb{C}^{N_{t} \times N_{t}}$ are Hermitian positive semi-definite matrices and satisfy $\sum_{k=1}^{L_{i}} \mathbf{Q}_{i, k} \succ \mathbf{0}_{N_{t}}$ (to have constraints on the power in all spatial directions).

\section{A. User Performance}

The performance of the $j$ th user in the $i$ th cell is measured by a strictly increasing continuous function $g_{i, j}\left(\operatorname{SINR}_{i, j}\right)$ of the SINR, which is defined as

$$
\operatorname{SINR}_{i, j}=\frac{\left|\mathbf{h}_{i, i, j}^{H} \mathbf{w}_{i, j}\right|^{2}}{\sum_{l \neq j}\left|\mathbf{h}_{i, i, j}^{H} \mathbf{w}_{i, l}\right|^{2}+\sum_{m \neq i}\left\|\mathbf{h}_{m, i, j}^{H} \mathbf{W}_{m}\right\|_{2}^{2}+\sigma^{2}} .
$$

and is a function of all $\mathbf{W}_{m}$, although not written explicitly. We let $g_{i, j}(0)=0$ and thus good performance means large positive values on $g_{i, j}\left(\mathrm{SINR}_{i, j}\right)$. However, its exact value cannot be calculated and optimized under CSI uncertainty. Instead, we consider the worst-case user performance

$$
\begin{aligned}
\min _{m, i, j} \in \mathcal{U}_{m, i, j} \forall m \neq i & g_{i, j}\left(\operatorname{SINR}_{i, j}\right) \\
= & g_{i, j}(\underbrace{\underbrace{}_{\mathbf{h}_{m, i, j} \in \mathcal{U}_{m, i, j} \forall m \neq i} \operatorname{SINR}_{i, j}}_{=\widetilde{\operatorname{SINR}}_{i, j}})
\end{aligned}
$$

where the equality follows from that $g_{i, j}(\cdot)$ is a strictly increasing function. We will use $\widetilde{\operatorname{SINR}}_{i, j}$ to denote the worstcase SINR in the remainder of the paper.

The user performance is limited by both power constraints and co-user interference. Under worst-case robustness, this relationship is characterized by the robust performance region: Definition 1. The robust performance region $\mathcal{R} \subset \mathbb{R}_{+}^{N K}$ is

$\mathcal{R}=\left\{\left(g_{1,1}\left(\widetilde{\operatorname{SINR}}_{1,1}\right), \ldots, g_{N, K}\left(\widetilde{\operatorname{SINR}}_{N, K}\right)\right): \mathbf{W}_{i} \in \mathcal{W}_{i} \forall i\right\}$

where the set of feasible beamforming matrices in the $i$ th cell is

$$
\mathcal{W}_{i}=\left\{\mathbf{W}_{i}: \operatorname{tr}\left\{\mathbf{W}_{i}^{H} \mathbf{Q}_{i, k} \mathbf{W}_{i}\right\} \leq q_{i, k} \forall k\right\}
$$

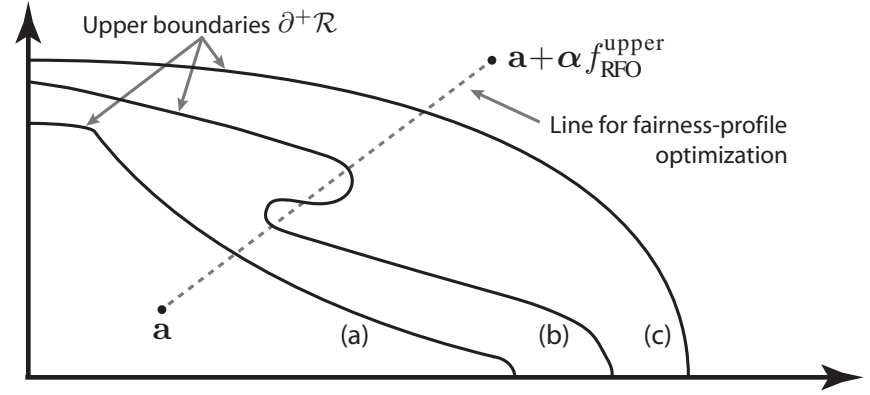

Fig. 2. Examples of regions with different shapes: (a) is normal but nonconvex, (b) is neither normal nor convex, and (c) is both normal and convex. Simple bisection along a fairness-profile is not guaranteed to find the upper boundary of non-normal regions, but fortunately $\mathcal{R}$ is a normal region.

This region has the dimension $N K$ of the total number of users (any user ordering can be used) and describes the combinations of performance that is guaranteed to be simultaneously achievable by the different users. In general, it is a non-convex set but it is easy to prove that $\mathcal{R}$ is compact and normal. ${ }^{1}$ For later use, we let $\partial^{+} \mathcal{R}$ denote the upper boundary of $\mathcal{R}$. Possible and impossible shapes of $\mathcal{R}$ are illustrated in Fig. 2, along with the corresponding upper boundaries.

\section{B. System Performance}

The system performance is measured by a function $f: \mathcal{R} \rightarrow$ $\mathbb{R}$ that takes points in the robust performance region $\mathcal{R}$ and produces scalar values. For a given $\mathbf{g} \in \mathcal{R}$, examples are

- Sum performance: $f(\mathbf{g})=\sum_{i, j} g_{i, j}$;

- Proportional fairness: $f(\mathbf{g})=\prod_{i, j} g_{i, j}^{1 /(N K)}$;

- Harmonic mean: $f(\mathbf{g})=N K\left(\sum_{i, j} g_{i, j}^{-1}\right)^{-1}$;

- Max-min fairness: $f(\mathbf{g})=\min _{i, j} g_{i, j}$.

Herein we consider any performance function $f(\cdot)$ that is Lipschitz continuous and strictly increasing, and thus we will solve the multicell optimization problem

$$
\underset{\mathbf{g} \in \mathcal{R}}{\operatorname{maximize}} f(\mathbf{g}) \text {. }
$$

Apparently, system optimization corresponds to a search in the robust performance region. We have the following result [7]:

Lemma 1. The global optimum to (9) is attained on the upper boundary $\partial^{+} \mathcal{R}$ of the robust performance region $\mathcal{R}$.

Thus, we only need to search the upper boundary to solve (9), but this is not as easy as it seems since it is hard to characterize $\partial^{+} \mathcal{R}$; even under perfect CSI the problem is NPhard for most performance functions $f(\cdot)$ [1]. However, the algorithm proposed in Section III still solves (9) optimally, but with a complexity unsuitable for real-time applications. In Section IV, we use the optimal solution for benchmarking.

\section{Robust MonOtOniC Optimization FramewORK}

In this section, we propose an algorithm for solving the optimization problem in (9) with global convergence.

${ }^{1}$ A set $\mathcal{T} \subset \mathbb{R}_{+}^{n}$ is called normal if for any point $\mathbf{x} \in \mathcal{T}$, all $\mathbf{x}^{\prime} \in \mathbb{R}_{+}^{n}$ with $\mathbf{x}^{\prime} \leq \mathbf{x}$ also satisfy $\mathbf{x}^{\prime} \in \mathcal{T}$. Under perfect CSI, this natural property can be proved for $\mathcal{R}$ by fixing the beamforming directions $\mathbf{w}_{i, j} /\left\|\mathbf{w}_{i, j}\right\|_{2}$ for a strategy that achieves $\mathbf{x}$ and showing that any $\mathbf{x}^{\prime} \leq \mathbf{x}$ can be achieved by changing the power allocation. See [7] for a proof under channel uncertainty. 


\section{A. Robust Fairness-Profile Optimization (RFO)}

First, we consider the following special case of the problem in (9) that we call robust fairness-profile optimization (RFO):

$$
\begin{aligned}
\underset{\mathbf{W}_{i} \in \mathcal{W}_{i} \forall i}{\operatorname{maximize}} & \min _{i, j} \frac{g_{i, j}\left(\widetilde{\operatorname{SINR}}_{i, j}\right)-a_{i, j}}{\alpha_{i, j}} \\
\text { s.t. } & g_{i, j}\left(\widetilde{\operatorname{SINR}}_{i, j}\right) \geq a_{i, j} \quad \forall i, j .
\end{aligned}
$$

This is a generalization of classic max-min optimization (typically solved by bisection [10]) and has two fairness constraints: 1) Each user has a lowest acceptable performance level $g_{i, j} \geq a_{i, j} \geq 0$;2) Each user gets a predefined portion $\alpha_{i, j} \geq 0$ of the exceeding performance (with $\sum_{i, j} \alpha_{i, j}=1$ ). Obviously, the RFO problem is infeasible if $a_{i, j}$ is too large. Despite these generalizations and that users can have different performance measures $g_{i, j}(\cdot),(10)$ can be solved by bisection: Lemma 2. For given $a_{i, j}, \alpha_{i, j}$ and an upper bound $f_{\mathrm{RFO}}^{\text {upper }}$ on optimum of (10), the problem can be solved by bisection over $\mathcal{F}=\left[0, f_{\mathrm{RFO}}^{\text {upper }}\right]$. For given $f_{\text {candidate }} \in \mathcal{F}$, the feasibility problem

$$
\begin{array}{ll}
\text { find } & \mathbf{W}_{i} \in \mathcal{W}_{i} \quad \forall i \\
\text { s.t. } & \widetilde{\operatorname{SINR}}_{i, j} \geq \gamma_{i, j} \quad \forall i, j,
\end{array}
$$

is solved for $\gamma_{i, j}=g_{i, j}^{-1}\left(a_{i, j}+\alpha_{i, j} f_{\text {candidate }}\right)$. If the problem is feasible, all $\tilde{f} \in \mathcal{F}$ with $\tilde{f}<f_{\text {candidate }}$ are removed. Otherwise, all $\tilde{f} \in \mathcal{F}$ with $\tilde{f} \geq f_{\text {candidate }}$ are removed. The initial feasibility of (10) is checked by solving (11) for $f_{\text {candidate }}=0$.

An initial upper bound $f_{\mathrm{RFO}}^{\text {upper }}$ is simple to achieve by relaxing the problem (e.g., by ignoring all interference); see [7]. The proposed bisection algorithm searches for the solution on a line segment in the robust performance region; see Fig. 2. This approach is useful since it solves (10) as a series of feasibility problems, which actually are convex:

Theorem 1. With uncertain inter-cell CSI as defined in (3), the feasibility problem in (11) is equivalent to the convex problem

$$
\begin{aligned}
& \text { find } \mathbf{W}_{i} \in \mathcal{W}_{i}, \lambda_{m, i, j} \geq 0, b_{m, i, j} \geq 0 \forall i, j, m \neq i \\
& \text { s.t. }\left[\begin{array}{ccc}
b_{m, i, j}-\lambda_{m, i, j} \widehat{\mathbf{h}}_{m, i, j}^{H} \mathbf{W}_{m} & \mathbf{0} \\
\mathbf{W}_{m}^{H} \widehat{\mathbf{h}}_{m, i, j} & b_{m, i, j} \mathbf{I}_{K} & \mathbf{W}_{m}^{H} \mathbf{B}_{m, i, j} \\
\mathbf{0} & \mathbf{B}_{m, i, j}^{H} \mathbf{W}_{m} & \lambda_{m, i, j} \mathbf{I}_{N_{t}}
\end{array}\right] \succeq \mathbf{0} \quad \forall i, j, m \neq i \\
& \sqrt{1+\frac{1}{\gamma_{i, j}}} \mathbf{h}_{i, i, j}^{H} \mathbf{w}_{i, j} \geq \sqrt{\left\|\mathbf{h}_{i, i, j}^{H} \mathbf{W}_{i}\right\|_{2}^{2}+\sum_{m \neq i} b_{m, i, j}^{2}+\sigma^{2}} \forall i, j .
\end{aligned}
$$

Proof: Under perfect CSI, the SINR constraints of (11) can be written as convex second-order cones as in the last constraint of (12) with $b_{m, i, j}=\left\|\mathbf{h}_{m, i, j}^{H} \mathbf{W}_{m}\right\|_{2}$ [8]. By introducing $b_{m, i, j}$ as an extra variable and adding new constraints

$$
b_{m, i, j} \geq \max _{\mathbf{h}_{m, i, j} \in \mathcal{U}_{m, i, j}}\left\|\mathbf{h}_{m, i, j}^{H} \mathbf{W}_{m}\right\|_{2},
$$

the SINR constraint will retain this convex structure under uncertain CSI. Next, we apply the Schur complement lemma and the uncertainty definition in (3) to rewrite (13) as

$$
\left[\begin{array}{cc}
b_{m, i, j} & \left.\left(\widehat{\mathbf{h}}_{m, i, j}^{H}+\tilde{\boldsymbol{\epsilon}}_{m, i, j}^{H} \mathbf{B}_{m, i, j}^{H}\right) \mathbf{W}_{m}\right] \\
\mathbf{W}_{m}^{H}\left(\widehat{\mathbf{h}}_{m, i, j}+\mathbf{B}_{m, i, j} \tilde{\boldsymbol{\epsilon}}_{m, i, j}\right) & b_{m, i, j} \\
\forall \tilde{\boldsymbol{\epsilon}}_{m, i, j} \text { with }\left\|\tilde{\boldsymbol{\epsilon}}_{m, i, j}\right\|_{2} \leq 1 .
\end{array}\right.
$$

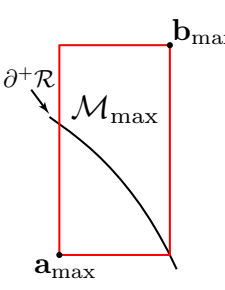

Selected box

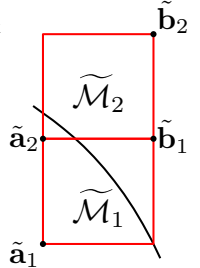

Branching

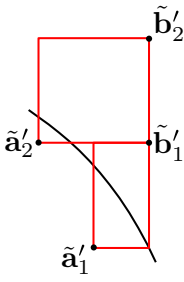

Reduction

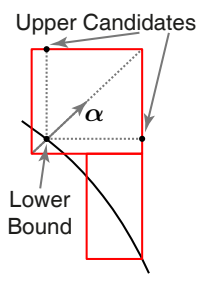

Bounding
Fig. 3. An iteration of the BRB algorithm: A box is selected and branched into two new boxes. These are reduced based on bounds on the solution. Finally, line search in the outmost box is used to improve the bounds.

Finally, we apply the well-known S-lemma from worst-case robustness theory (see [7], [9], [10]) to rewrite (14) as the convex semi-definite constraint in (12).

The conclusion is that RFO problems constitute a special class of robust system optimization problems that are quasiconvex and thus can be solved globally in polynomial time. We cannot expect this structure for general monotonic functions $f(\cdot)$, but the next subsection proposes an optimization framework that solves (9) by iteratively solving RFO problems.

\section{B. Branch-Reduce-Bound (BRB) Algorithm}

Next, we propose a branch-reduce-and-bound (BRB) algorithm for solving any robust monotonic optimization problem in (9) with global convergence. It is based on the generic BRB algorithm in [2]. Herein, we provide an outline of our multicell beamforming version, while all the details are available in [7].

The algorithm maintains a set $\mathcal{N}$ with non-overlapping boxes $^{2}$ that surely covers the part of the robust performance region $\mathcal{R}$ where the optimal solutions lie. Iteratively, we split boxes and try to improve a lower bound $f_{\min }$ and upper bound $f_{\max }$ on the optimum of (9). The algorithm proceeds until $f_{\max }-f_{\min }<\varepsilon$, for some predefined solution accuracy $\varepsilon$.

Initially, $\mathcal{N}=\left\{\mathcal{M}_{0}\right\}$ for a box $\mathcal{M}_{0}=\left[\mathbf{0}, \mathbf{b}_{0}\right] \subset \mathbb{R}_{+}^{N K}$ where $\mathbf{b}_{0}$ is based on some suitable upper bound that guarantees $\mathcal{R} \subseteq \mathcal{M}_{0}$ (see [7]). The initial lower and upper bounds can be taken as $f_{\min }=f(\mathbf{0})=0$ and $f_{\max }=f\left(\mathbf{b}_{0}\right)$.

Each iteration of the algorithm consists of three steps: 1) branching; 2) reduction; and 3) bounding. These steps are outlined below and are illustrated in Fig. 3 .

1) Branching: Each box $\mathcal{M} \in \mathcal{N}$ has a local upper bound, denoted $\beta(\mathcal{M})$. At least one $\mathcal{M}_{\max }=\left[\mathbf{a}_{\max }, \mathbf{b}_{\max }\right] \in \mathcal{N}$ will have $\beta\left(\mathcal{M}_{\max }\right)=f_{\max }$. Each iteration begins with selecting this box and cutting it into two new boxes $\widetilde{\mathcal{M}}_{1}, \widetilde{\mathcal{M}}_{2}$ by bisecting $\mathcal{M}_{\max }$ along the longest side. The (local) upper bounds for these boxes are the minimum of $\beta\left(\mathcal{M}_{\max }\right)$ and the function value at each box's upper corner.

2) Reduction: Next, the new boxes $\widetilde{\mathcal{M}}_{k}=\left[\tilde{\mathbf{a}}_{k}, \tilde{\mathbf{b}}_{k}\right], k=$ 1,2 , are reduced by cutting off parts that cannot achieve function values between the lower bound $f_{\min }$ and (local) upper bound $\beta\left(\widetilde{\mathcal{M}}_{k}\right)$. The whole box is removed from $\mathcal{N}$ if $\beta\left(\widetilde{\mathcal{M}}_{k}\right)<f_{\min }$, but usually it is replaced by a (potentially) smaller box $\left[\tilde{\mathbf{a}}_{k}^{\prime}, \tilde{\mathbf{b}}_{k}^{\prime}\right]$. While the reduction principle is simple, the mathematical definition is messy and is given in [7].

\footnotetext{
${ }^{2} \mathrm{~A}$ box $[\mathbf{a}, \mathbf{b}] \in \mathbb{R}_{+}^{N K}$ contains all $\mathbf{x} \in \mathbb{R}_{+}^{N K}$ such that $\mathbf{a} \leq \mathbf{x} \leq \mathbf{b}$.
} 


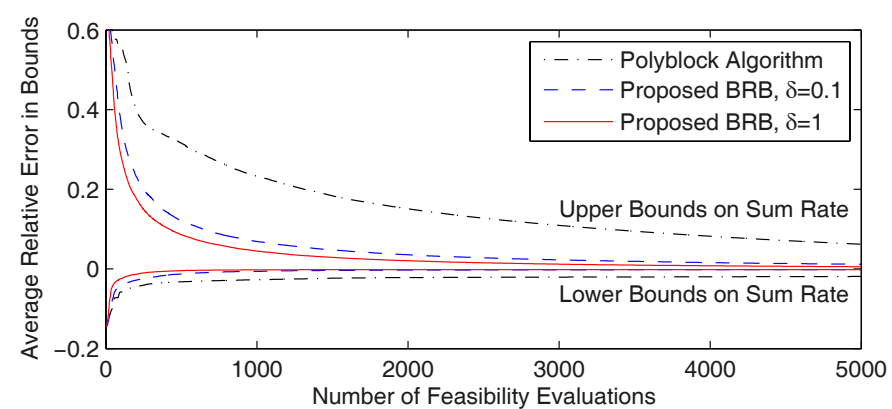

Fig. 4. Relative error of lower and upper bounds on the sum rate.

3) Bounding: Each iteration ends with an attempt to improve the bounds $f_{\min }, f_{\max }$. For this purpose, we do a linesearch from the lower to the upper corner of the outmost box $\widetilde{\mathcal{M}}_{2}=\left[\tilde{\mathbf{a}}_{2}^{\prime}, \tilde{\mathbf{b}}_{2}^{\prime}\right]$, which can be formulated as a RFO problem: Lemma 3. The highest feasible performance in $\mathcal{M}=[\mathbf{a}, \mathbf{b}]$ can be bounded as $\left[\bar{f}_{\min }, \bar{f}_{\text {max }}\right]$ for

$$
\begin{aligned}
& \bar{f}_{\min }=f\left(\mathbf{a}+\boldsymbol{\alpha} f_{\mathrm{RFO}}^{\min }\right) \\
& \bar{f}_{\max }=\max _{1 \leq k \leq N} f\left(\mathbf{b}-\left(b_{k}-n_{k}\right) \mathbf{e}_{k}\right)
\end{aligned}
$$

where $\mathbf{n}=\mathbf{a}+\boldsymbol{\alpha} f_{\mathrm{RFO}}^{\max }, \boldsymbol{\alpha}=(\mathbf{b}-\mathbf{a}) /\|\mathbf{b}-\mathbf{a}\|_{1}$, and $\mathbf{e}_{k}$ denotes the $k$ th column of an $N K$-dimensional identity matrix. The variables $f_{\mathrm{RFO}}^{\min }, f_{\mathrm{RFO}}^{\max }$ are the interval endpoints achieved by Lemma 2 with starting-point $\mathbf{a}$, direction $\boldsymbol{\alpha}=(\mathbf{b}-\mathbf{a}) / \| \mathbf{b}-$ $\mathbf{a}\left\|_{1}, f_{\mathrm{RFO}}^{\mathrm{upper}}=\right\| \mathbf{b}-\mathbf{a} \|_{1}$, and some line-search accuracy $\delta$.

We use the values on $\bar{f}_{\min }, \bar{f}_{\max }$ to update our bounds:

1) Replace $f_{\min }$ with $\bar{f}_{\min }$ if $\bar{f}_{\min } \geq f_{\min }$;

2) Set $\beta\left(\widetilde{\mathcal{M}}_{2}\right)=\bar{f}_{\max }$ if $\bar{f}_{\max }<\beta\left(\overline{\mathcal{M}}_{2}\right)$;

3) Set $f_{\max }=\max _{\mathcal{M} \in \mathcal{N}} \beta(\mathcal{M})$.

\section{Global Convergence of the BRB Algorithm}

If $f_{\text {opt }}$ is the global optimum, the BRB algorithm finds an interval $f_{\text {opt }} \in\left[f_{\min }, f_{\max }\right]$ that satisfies $f_{\max }-f_{\min }<\varepsilon$ in finitely many iterations. The formal proof is given in [7] and it can be noted that any line-search accuracy $\delta>0$ can be used. Another remark is that although the algorithm converges, the worst-case speed of this NP-hard problem is exponential in $K$. But we will show that the BRB algorithm is still very useful for benchmarking of suboptimal beamforming strategies.

\section{Numerical Evaluation AND Discussion}

In the numerical evaluation, we have $N=2$ cells and $K=$ 2 users per cell. The total transmit power per base station is constrained with $L_{i}=1, \mathbf{Q}_{i, 1}=\mathbf{I}_{N_{t}}$, and where $q_{i, 1} / \sigma^{2}$ is the transmit SNR. We generate the exact intracells channel as $\mathbf{h}_{i, i, j} \in \mathcal{C N}\left(\mathbf{0}, \mathbf{I}_{N_{t}}\right)$ and the uncertain intercell channels as $\widehat{\mathbf{h}}_{m, i, j} \in \mathcal{C N}\left(\mathbf{0}, \frac{1}{2} \mathbf{I}_{N_{t}}\right), m \neq i$. Spherical uncertainty sets are assumed with $\mathbf{B}_{m, i, j}=\sqrt{\xi} \mathbf{I}_{N_{t}}$, where $\sqrt{\xi}$ is the radius.

First, we study the convergence of the BRB algorithm for $N_{t}=3$ and a transmit SNR of $20 \mathrm{~dB}$. To compare with the polyblock approximation (PA) algorithm in [5], we let $\xi=0$. The main complexity of both algorithms originates from solving feasibility problems of the type in (11), thus the number of evaluations is on the horizontal axis of Fig. 4. This figure shows the average relative deviations of the lower and upper

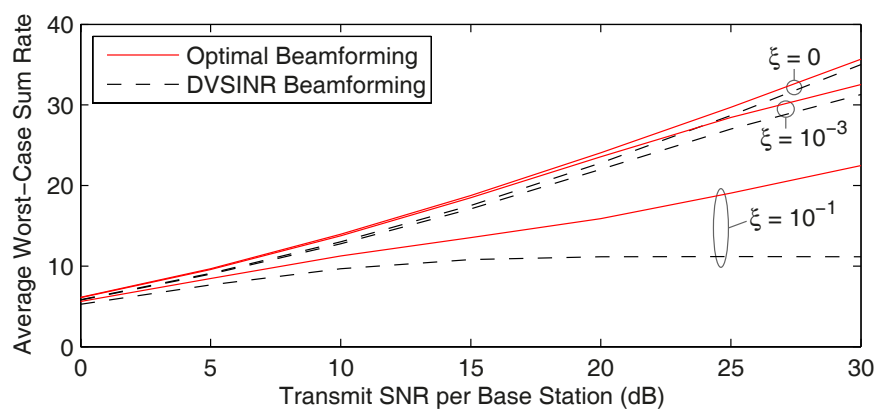

Fig. 5. Worst-case sum rate with optimal and DVSINR beamforming.

bound on the sum rate, $\left(f_{\min }-f_{\text {opt }}\right) / f_{\text {opt }}$ and $\left(f_{\max }-f_{\text {opt }}\right) / f_{\text {opt }}$, respectively, for different line-search accuracies. Clearly, the proposed BRB algorithm has better convergence than the PA algorithm, which is consistent with similar observations in [2]. Note that both algorithms find quite good lower bounds after relatively few evaluations, while it takes much longer time for PA to achieve tight upper bounds than for BRB.

Finally, we demonstrate that our framework can benchmark heuristic beamforming strategies under uncertain CSI. In Fig. 5, the average worst-case sum rate is given with optimal beamforming (calculated using the BRB algorithm) and with DVSINR beamforming proposed in [12] (we have $N_{t}=4$ ). This distributed strategy performs quite well at low and at high SNR, and is robust to small errors (e.g., $\xi=10^{-3}$ ) but becomes highly suboptimal as the uncertainty increases.

\section{REFERENCES}

[1] Y.-F. Liu, Y.-H. Dai, and Z.-Q. Luo, "Coordinated beamforming for MISO interference channel: Complexity analysis and efficient algorithms," IEEE Trans. Signal Process., vol. 59, pp. 1142-1157, 2011.

[2] H. Tuy, F. Al-Khayyal, and P. Thach, "Monotonic optimization: Branch and cut methods," in Essays and Surveys in Global Optimization, C. Audet, P. Hansen, and G. Savard, Eds. Springer US, 2005.

[3] E. Jorswieck and E. Larsson, "Monotonic optimization framework for the two-user MISO interference channel," IEEE Trans. Commun., vol. 58, no. 7, pp. 2159-2168, 2010.

[4] K. Eriksson, S. Shi, N. Vučić, M. Schubert, and E. Larsson, "Globally optimal resource allocation for achieving maximum weighted sum rate," in Proc. GLOBECOM'10, 2010.

[5] J. Brehmer and W. Utschick, "Optimal interference management in multi-antenna, multi-cell systems," in Proc. Int. Zurich Seminar on Commun., 2010, pp. 134-137.

[6] M. Rossi, A. Tulino, O. Simeone, and A. Haimovich, "Non-convex utility maximization in Gaussian MISO broadcast and interference channels," in Proc. IEEE ICASSP'11, 2011, pp. 2960-2963.

[7] E. Björnson, G. Zheng, M. Bengtsson, and B. Ottersten, "Robust monotonic optimization framework for multicell MISO systems," IEEE Trans. Signal Process., submitted in Mar 2011, arXiv:1104.5240v2.

[8] H. Dahrouj and W. Yu, "Coordinated beamforming for the multicell multi-antenna wireless system," IEEE Trans. Wireless Commun., vol. 9, no. 5, pp. 1748-1759, 2010.

[9] N. Vučić and H. Boche, "Robust QoS-constrained optimization of downlink multiuser MISO systems," IEEE Trans. Signal Process., vol. 57, no. 2, pp. 714-725, 2009.

[10] M. B. Shenouda and T. Davidson, "Nonlinear and linear broadcasting with QoS requirements: Tractable approaches for bounded channel uncertainties," IEEE Trans. Signal Process., vol. 57, no. 5, 2009.

[11] K.-Y. Wang, T.-H. Chang, and C.-Y. C. W.-K. Ma, A. So, "Probabilistic SINR constrained robust transmit beamforming: A Bernstein-type inequality based conservative approach," in Proc. IEEE ICASSP' 11, 2011.

[12] E. Björnson, R. Zakhour, D. Gesbert, and B. Ottersten, "Cooperative multicell precoding: Rate region characterization and distributed strategies with instantaneous and statistical CSI," IEEE Trans. Signal Process., vol. 58, no. 8, pp. 4298-4310, 2010. 